\title{
On the neuronal basis for multisensory convergence: a brief overview
}

\author{
M. Alex Meredith* \\ Department of Anatomy, Visual/Motor Neuroscience Division, Virginia Commonwealth University School of Medicine, Richmond, VA 23298, USA
}

Accepted 22 May 2001

\begin{abstract}
For multisensory stimulation to effect perceptual and behavioral responses, information from the different sensory systems must converge on individual neurons. A great deal is already known regarding processing within the separate sensory systems, as well as about many of the integrative and perceptual/behavioral effects of multisensory processing. However, virtually nothing is known about the functional architecture that underlies multisensory convergence even though it is an integral step to this processing sequence. This paper seeks to summarize the findings pertinent to multisensory convergence, and to initiate the identification of specific convergence patterns that may underlie different multisensory perceptual and behavioral effects. (C) 2002 Elsevier Science B.V. All rights reserved.
\end{abstract}

Keywords: Multisensory; Association cortex; Superior colliculus; Excitation; Inhibition; Neural circuits

\section{Introduction}

The nervous system extracts information from environmental events and converts it to perceptions, memories, and/or behaviors. A great deal is known regarding how this processing occurs within individual sensory channels, such as vision, somatosensation, or audition. However, environmental events are often detected by two or more different sensory systems at the same time, and the perceptual and behavioral consequences of multisensory stimulation are often quite distinct from those evoked by either sensory component alone. Numerous multisensory behavioral and perceptual effects have been described, ranging from escape, detection, identification and orientation to cognitive development, postural control, tool use and language perception. The neuronal bases for some of these effects have been examined and the factors governing specific multisensory behaviors (e.g., orienting) have been shown to apply to the activity of multisensory neurons as well [52]. At the neuronal level, responses to multiple sensory stimuli result in a measure of integrated activity that no longer resembles the discharges evoked by either of the modality-specific components alone. The resultant form of multisensory integration (either response enhancement or response depression) is predictable according to the spatial and temporal relationships of the stimuli

*Tel.: +1-804-828-9533; fax: +1-804-828-9477.

E-mail address: mameredi@hsc.vcu.edu (M.A. Meredith). as well as their physical parameters (described below, also see Refs. [37,39,40,45,56]). However, for multisensory integration to occur and for it to ultimately lead to distinct perceptual or behavioral effects, information must first converge from the different sensory systems onto individual neurons (see Fig. 1). There is considerable evidence that multisensory convergence occurs widely throughout the neuroaxis and across phylogeny (for review, see Ref. [54]). Given its prevalence, it is surprising that very little is known regarding the organization, architecture, or circuitry that underlies multisensory convergence itself, especially within mammalian systems. Even for the best understood of mammalian multisensory neurons, which are those in the superior colliculus, the specific afferent sources, targets, and patterns of termination on identified multisensory neurons are currently known only in general terms (e.g., see Ref. [23]). Furthermore, it is unlikely that the synaptology, pharmacology, or cytoarchitecture of the superior colliculus represents the entire range of multisensory convergence patterns for the brain. Therefore, the goal of this overview is to initiate a search for the general principles of multisensory convergence. Discussed will be what is already known about multisensory convergence in relation to identified multisensory functions, as well as newly identified forms of multisensory convergence and their possible behavioral/ perceptual relevance and, finally, potential areas that hold promise for revealing an even broader range of functional architecture for multisensory convergence. Any assump- 


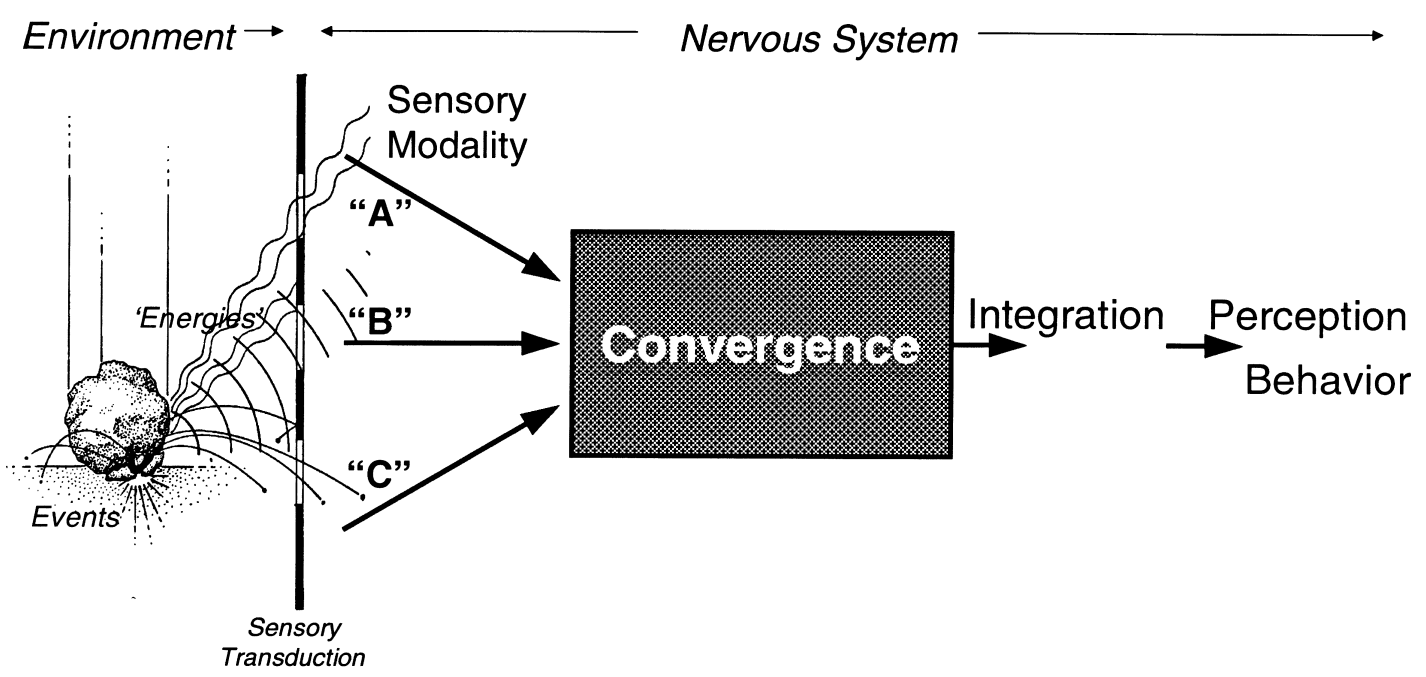

Fig. 1. The sequence of multisensory processing. At the left, environmental events frequently generate several simultaneous physical effects, or 'energies.' Each 'energy,' such as light, air-borne vibrations, or dispersion of particulate matter, radiates from the causal event without influencing the physical properties of the others. When these physical 'energies' are encountered by a nervous system, each is detected or transduced by receptors specifically tuned for a particular stimulus modality (e.g., retina for light, cochlea for air-borne vibrations, etc.). In essence, each receptor acts like a selective filter (gaps in vertical black bar) that responds only to one particular stimulus modality to the exclusion of the others, and these modality-specific sensory channels are largely preserved among their central projections. However, in numerous regions of the brain, modality-specific projections ('A', 'B' and 'C') have projections that converge onto individual neurons, creating those that are influenced by more than one sensory modality: multisensory neurons. The shaded box indicates that little is known about the functional architecture of multisensory convergence. However, once convergence occurs, multisensory integration can result in neuronal activity which leads to alterations in perception and or behavior that would not be predicted by responses to unimodal stimuli presented alone.

tions regarding convergence, however, must be preceded by the demonstration that the different input channels involved are, in fact, distinct and independent.

\section{The physical nature of environmental events}

Environmental events would pass undetected by the nervous system except for the physical 'energies' they produce (or reflect) that are detectable as sensory stimuli. These 'energies' can occur in a myriad of forms, but the most familiar are those which occur as light (within specific electromagnetic wavelengths), air-borne vibrations, solid-body vibrations, or the dispersion of air- or water-borne molecules. Each of these 'energies' has its own physical properties, such as its velocity of propagation, and there is virtually no cross-talk between the physical attributes of these different effects. For example, vibrations will progress until dissipated whether in the presence of light or not.

Events often happen, like the impact of a falling boulder, that simultaneously generate several different 'energies,' as schematized in Fig. 1. In this example, the crashing stone not only reflects light at the moment the rock strikes the ground, but also creates air-borne and ground-borne vibrations and scatters particulate matter into the air. Because of their different physical nature, each of these 'energies' has no effect on the other. Yet some of these physical 'energies' can be transduced by the nervous system, and the transduced 'energy' is defined as a stimulus. A 'multisensory stimulus' is actually, then, an event which generates several independent physical 'energies' each of which is simultaneously detectable by different types of sensory receptors. Accordingly, the multisensory status of an event has as much to do with the design of the recipient nervous system as the physical event itself.

\section{Sensory transduction: from physical stimulus to receptor-specific signal}

The different stimulus modalities, such as light, vibration, air-borne or solubilized chemicals, are transduced by specialized receptors into neural signals. Each receptor is 'tuned' to a specific range of stimuli. The mammalian retina transduces light within the spectrum of wavelengths referred to as 'white' light, the cochlea transduces airborne pressure waves within frequencies of approximately $50-45,000 \mathrm{~Hz}$, and nerve endings in the skin signal forces impinging upon them. Some animals exhibit adaptive specializations that either expand the range in which these receptor organs operate, or alter their relative roles in perception and behavior. For example, dogs and cats hear at higher frequency ranges than humans; bats, whales and dolphins have entire sensory repertoires dedicated to ultrasonic perception. In addition, rodents are dependent on highly modified facial hairs, called vibrissae, to direct a 
variety of behaviors, while some reptiles, such as rattlesnakes, augment their sensory repertoire with pit organs for the detection of infrared signals [22].

Each of the different sensory receptors produce signals that lead through sensory-specific projection pathways to result in distinct perceptual experiences. For example, there is simply no commonality between the color blue, the musical key of ' $G$ ', and a pinch. Such modality-specific sensory perceptions have, no doubt, been enhanced by the elaboration of sensory cortices in complex nervous systems, which allow for progressively higher levels of feature extraction and processing. Also, a specific environmental event can be perceived very differently by different species depending on their own particular array of sensory receptors. For example, we as humans perceive riding next to an open car window largely as an auditory and tactile experience, whereas for dogs the incoming wind no doubt also carries a rushing riot of odors.

\section{Multisensory convergence}

In contrast to the organization of the modality-specific pathways, the neural connections from the different receptor organs must find their way onto shared neurons for information from the different sensory systems to influence one another. At the point of convergence, a fundamental change occurs. Where previously there was no physical interaction between environmental stimuli or modalityspecific receptors, there now arises the potential for responses to one mode of 'energy' to be modified by responses elicited by another. Thus, by means of multisensory convergence, the resultant forms of processing represent an emergent property of the brain.
Numerous studies have provided anatomical or EEG/ ERP evidence for 'convergence' of inputs from different sensory modalities into a specific region [3,14,29,51]. However, unless functional interactions are observed, conclusions regarding convergence using these techniques must be rendered cautiously. As illustrated in Fig. 2, convergence within a particular region (areal convergence) does not prove that the convergence onto individual neurons (neuronal convergence) necessary for multisensory integration has occurred. Instead, it may be possible for populations of different unimodal neurons to intermingle with one another within the same region (areal convergence) without sharing modality-related information. Anatomical markers that distinguish between unimodal and multisensory neurons would easily clarify such a distribution pattern, and are apparently under development [67]. However, among the current anatomical repertoire, only double-labelling electron microscopic techniques can provide reliable data regarding multisensory convergence. Such an examination would also provide a wealth of additional information about convergent synaptic forms, pre- and post-synaptic relationships, etc. Unfortunately, these ultrastructural studies have not yet been reported. At present the most reliable and efficient technique for identifying multisensory convergence is single-unit extracellular electrophysiological recording. Even so, relatively little is known about the nature of multisensory convergence onto individual neurons and the functional architecture underlying multisensory convergence anywhere in the mammalian brain has not yet been described. Instead, what is known about multisensory convergence in mammals has been inferred from the predominant model of multisensory integration, the superior colliculus (for an example, see Ref. [23]).

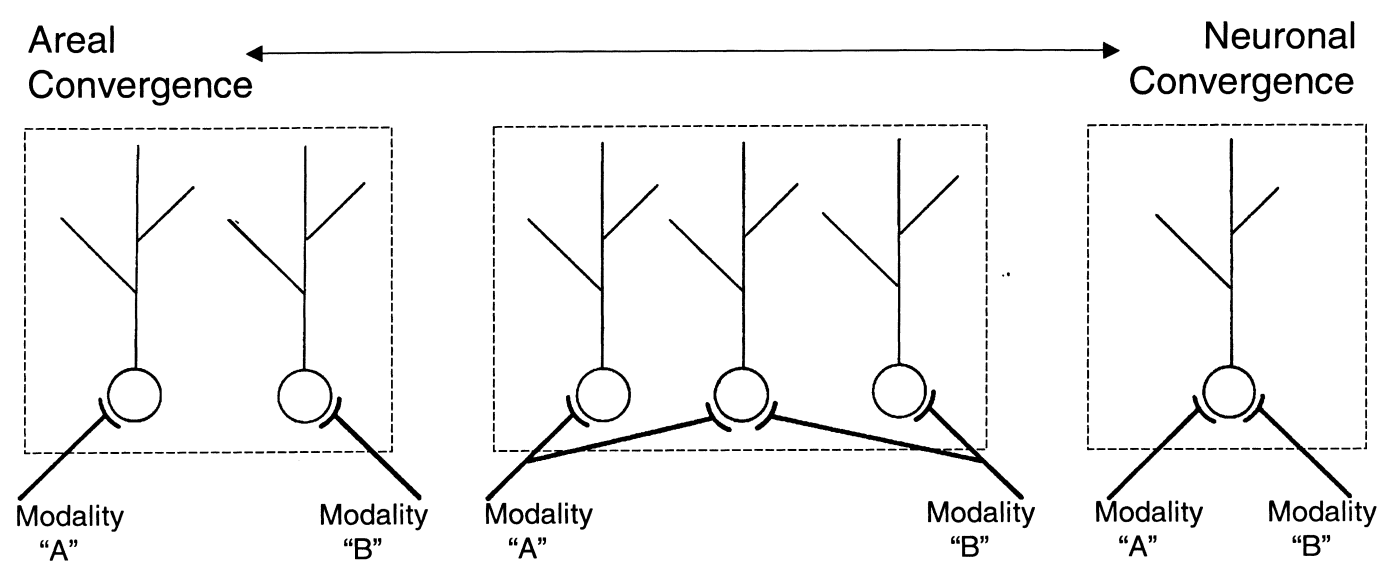

Fig. 2. Identifying multisensory convergence. Inputs from two different modalities ('A' and 'B') may converge within a given area (dashed box), but like the example on the left, the different inputs do not terminate on the same neuron (Areal Convergence). This form gives the anatomical appearance of convergence, but individual neurons respond only to one modality or the other and fail to integrate their simultaneous inputs. To the right, inputs from the different modalities converge onto the same neuron (Neuronal Convergence) and generate a multisensory neuron that not only responds to stimulation of either modality, but also can integrate their simultaneous inputs. In all likelihood, most neural areas related to multisensory functions fall within a continuum between these two forms and exhibit properties of both (see Center box). 


\section{Multisensory convergence: excitatory-excitatory}

The superior colliculus is a laminated midbrain structure that is composed of alternating fibrous and cellular layers. The upper three layers, commonly referred to as the superficial layers, receive inputs from the retina and primary visual cortex and contain a retinotopic map of visual space. The lower four layers, collectively termed the deep layers (combined intermediate and deepest laminae), also receive visual inputs but mostly from extraprimary visual cortical areas (for review, see Ref. [26]) that contribute to a coarse map of visual space there. Also found in the deep layers are maps of the body surface $[6,11,36,53,58]$ and auditory space $[32,41,65]$, which are constituted by afferents from the brainstem as well as from extraprimary sensory cortices $[23,26,35,60]$. Due to the convergence of these different excitatory inputs, many superior colliculus neurons can be activated by natural stimuli from more than one sensory modality. For example, the superior colliculus neuron depicted in Fig. 3 was excited by the presentation of a visual stimulus presented alone, as well as by an auditory stimulus presented alone. Therefore, by virtue of multisensory convergence, this neuron exhibits response properties of both sensory modalities. Depending on the species (see Table 1), a large proportion of deep layer superior colliculus neurons have been shown to receive excitatory inputs from more than one sensory modality. These types of multisensory neurons are commonly referred to as 'bimodal' (like that depicted in Fig. 3), while those which are activated by three modalities are 'trimodal.' Furthermore, because the dominant nature of sensory inputs to superior colliculus multisensory neurons is excitatory, they can also be described as exhibiting an 'excitatory-excitatory' form of multisensory convergence, as summarized schematically in Fig. 3D.

In addition to collicular involvement with multisensory processing, numerous multisensory perceptual effects have been documented as being cortically based (e.g., Refs.
Table 1

Proportion of multisensory neurons in deep layers of the superior colliculus, by species

\begin{tabular}{lc}
\hline Species & Percentage \\
\hline Monkey (Macacca mulatta) [55] & $<27 \%$ \\
Cat [47] & $55 \%$ \\
Ferret [29] & $22-50 \%$ \\
Hamster [5,51] & $<10 \%$ \\
\hline
\end{tabular}

$[25,27,49])$. In addition, single unit recording in the cortex, subcortex, and thalamus of primates (e.g., Refs. $[12,19,20,50])$, cats (e.g., Refs. $[2,24,28,59,64])$, and rodents (e.g., Ref. [46]) have revealed the presence of bimodal and trimodal neurons. Many of the cortical multisensory neurons respond to inputs from more than one sensory modality alone, show multisensory response enhancement when the stimuli are appropriately combined [59], and are also likely to represent a form of excitatoryexcitatory convergence similar to that described for collicular neurons.

When inputs from different sensory modalities simultaneously converge, many (but not all, see Ref. [30]) bimodal neurons integrate the responses in a fashion that no longer resembles that evoked by either of the sensory inputs alone. For example, the neuron depicted in Fig. 3 responded weakly to the presentation of an auditory stimulus ( $=2.6 \pm 1.9$ spikes/presentation), more robustly to the visual stimulus $(=12.6 \pm 51.9$ spikes/presentation $)$ and very strongly to their combination $(=26.8 \pm 7.5$ spikes/ presentation). In this case, the combination of stimuli evoked a response that was significantly $(P>0.05$, paired $t$-test) greater than the most vigorous unimodal response. This form of response integration has been termed multisensory enhancement [39]. Enhancement, however, is not simply a default mode for multisensory responses, but is dependent on the complex relationship of a variety of factors. The temporal relationship of the different stimuli is critical for the elicitation of response enhancement, and it

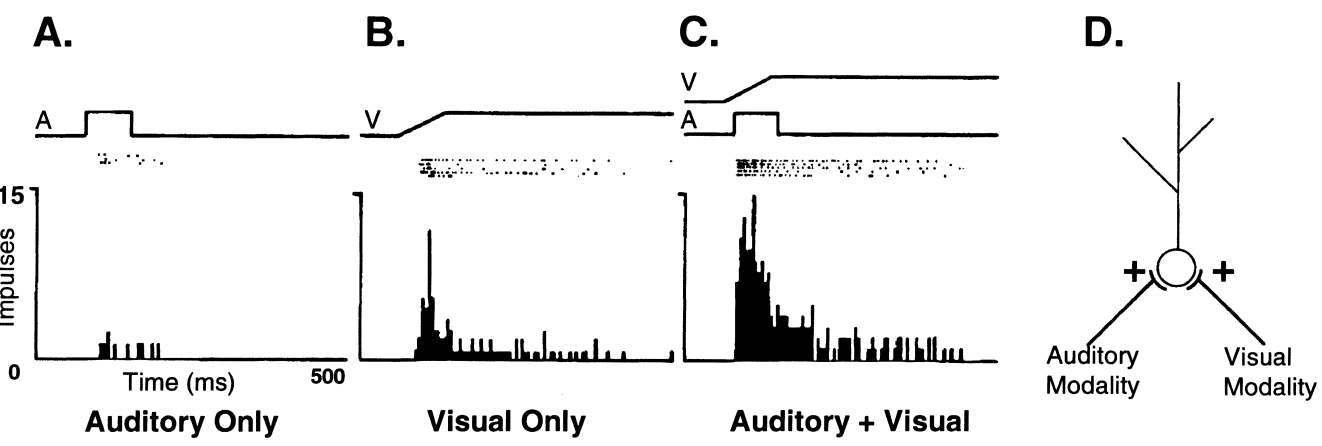

Fig. 3. The result of 'Excitatory-Excitatory' multisensory neuronal convergence. Extracellular recording from a cat superior colliculus neuron shows the responses of a neuron to individual stimulus presentations (rasters) as well as their summed activity (histograms, 10 ms bins) to (A) an auditory stimulus (squarewave labeled 'A'; white noise), (B) a visual stimulus (ramp labeled ' $\mathrm{V}$ '; bar of white light moved through visual receptive field) and (C) to the combination of the same auditory and visual stimuli. This neuron responded to either stimulus presented alone (A, B) as well as integrated their simultaneous inputs (C) in the form of response enhancement. Thus, the inputs from these different modalities were excitatory and, for this and other bimodal (and trimodal) neurons, represent an 'excitatory-excitatory' form (D) of multisensory convergence. 
has been shown that stimulus combinations that occur within $100 \mathrm{~ms}$ of one another have the highest likelihood of evoking such a response amplification [37]. This makes obvious sense because stimuli that occur in close temporal proximity are most likely to be initiated by the same environmental event. The spatial relationship of a stimulus combination is also critical for evoking multisensory response enhancement. Because the different receptive fields of a given multisensory neuron tend to exhibit a spatial correspondence $[31,39,40,62]$, those stimulus combinations that are aligned in space will fall within the excitatory receptive field areas and will generate response enhancement $[31,39,40]$. This also makes intuitive sense, since stimuli that arise from the same point in space are likely to be causally related and enhanced responses to their combination would be biologically adaptive. Ultimately, these temporal and spatial constraints act like a filter, or window, such as those illustrated in Fig. 4, through which only appropriately configured stimulus combinations are permitted access to circuitry leading to multisensory response amplification.

Other features that influence multisensory integration are the modality-specific response properties of the neuron(s) involved, such as directional or velocity preferences, intensity thresholds, etc. [56]. In addition, the relative level of stimulus effectiveness inversely influences the magnitude of multisensory response enhancement [39], such that stimuli that are maximally effective when presented alone often elicit little to no response amplification while those stimuli that are nearly undetectable by themselves tend to produce the highest levels of response enhancement. These factors are applicable not only to multisensory interactions in neurons of the superior colliculus, but also in those of the cerebral cortex and perhaps all other regions where excitatory-excitatory multisensory convergence has been documented [59]. Furthermore, these same factors governing multisensory integration at the neuronal level also apply to multisensory behaviors, such as detection and orienting [52,55].

\section{Multisensory convergence: excitatory-inhibitory}

Such 'excitatory-excitatory' architecture for multisensory convergence, however, is inadequate to account for several perceptual phenomena. For example, suppression of responses to a non-attended stimulus during selective cross-modal attention $[16,25,57]$ cannot be attributed to spatial excitatory effects. Specifically: a subject cued to attend to a visual stimulus at a particular locus reveals a reduced response when an auditory stimulus is substituted at the same location. Thus, focussing attention to a particular sensory modality among many distinguishes the responses to that stimulus for preferential processing. Implicit to this scheme is the active inhibition of responses in the non-attended modalities, as has been demonstrated for distractor stimuli from within the same modality $[42,47,66]$. Thus, for selective attention processes, convergence of excitatory inputs from one modality with inhibitory inputs from another presents a more vigorous model than that offered by multisensory neurons as they are currently envisioned. Evidence for this 'new' form of 'excitatory-inhibitory' multisensory convergence has been described in cat association cortex [7,10].

Recent studies of the cortex surrounding the Anterior Ectosylvian Sulcus (AES) of the cat reveal that areal multisensory convergence occurs between the auditory FAES region and the somatosensory SIV area [10]. However, neurons within the somatosensory region have not been reported to exhibit the bimodal properties that would

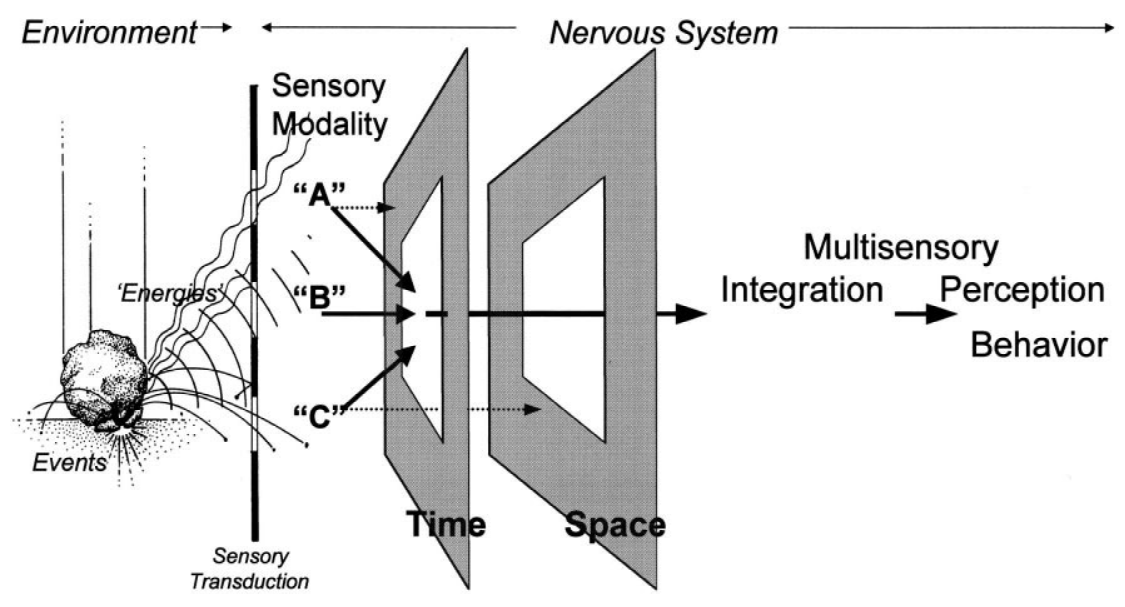

Fig. 4. Access to integrative multisensory circuits is determined by the spatio-temporal relationships of the stimuli. Environmental events, such as the impact of a falling boulder, release a multitude of physical energies that are transduced by different sensory receptors. However, for combinations of different stimuli ultimately to access integrative multisensory neural circuitry, they must occur within specific temporal (e.g., nearly at the same time) and spatial (e.g., nearly at the same place) windows. Events that occur outside these windows (stippled arrows) are processed as separate, modality-specific events. 


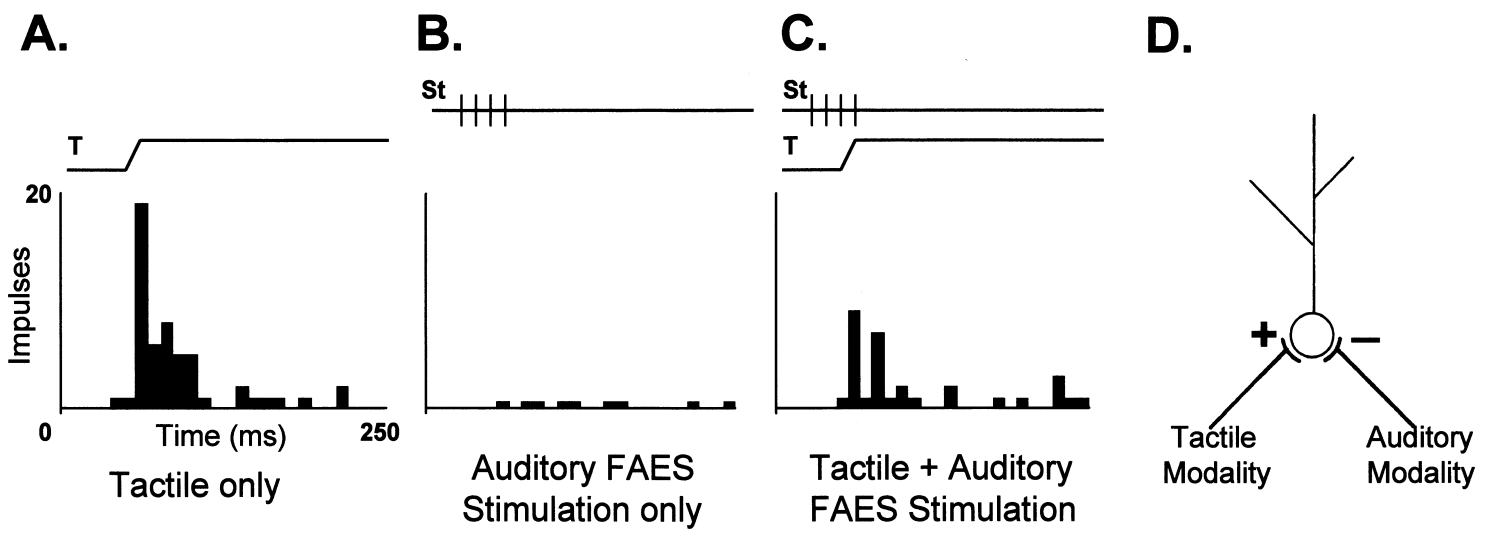

Fig. 5. The result of 'Excitatory-Inhibitory' multisensory neuronal convergence. Extracellular recording from a neuron in cat somatosensory cortical region SIV revealed responses to a tactile stimulus presented within the neuron's receptive field (A; represented by ramp labeled 'T'), but no activity was elicited in response to electrical stimulation of indwelling electrodes placed within the auditory cortical region, FAES (B; represented by the pulses labeled 'St'; $0.1 \mathrm{~ms}$ pulse, $100 \mathrm{~ms}$ train duration used at $100 \mathrm{~Hz}$ at $300 \mu \mathrm{A})$. However, when the same tactile and auditory cortical stimulation were combined (C), the neuronal response was suppressed. Because this neuron responded only to the tactile stimulus presented alone (A), and that response was diminished by the presence of auditory cortical stimulation (C), these data are consistent with an 'Excitatory-Inhibitory' form of multisensory convergence (D).

be expected if convergence occurred with excitatory auditory inputs $[8,9]$. Current studies assessing multisensory convergence in this region demonstrated that SIV neurons, tested specifically for responses to auditory cues presented alone, were not activated and, therefore, appeared to be purely somatosensory in nature [7]. In addition, when these same SIV neurons were tested for inputs from auditory FAES by electrical stimulation of the FAES, none were excited. However, when effective tactile stimulation was combined with electrical stimulation of the auditory FAES, the excitatory responses to the tactile stimulation of nearly $70 \%$ of the SIV neurons were significantly reduced, as illustrated in Fig. 5. Furthermore, preliminary experiments [7] show that the suppressive effect of auditory FAES stimulation on SIV somatosensory responses is blocked by the presence of Bicuculline methiodide, an antagonist to the inhibitory neurotransmitter GABA. These data indicate that the net result of somatosensory-auditory convergence within SIV is inhibitory and represents an 'excitatory-inhibitory' form of multisensory neuronal convergence (Fig. 5D).

In summary, there appear to be at least two forms of multisensory convergence occurring at the neuronal level. First, and most familiar, are those neurons which are overtly excited by stimuli from more than one modality. These bimodal (and trimodal) neurons result from an 'excitatory-excitatory' form of convergence, which is diagrammed in Fig. 6A. However, it is unlikely that any excitatory system would be left unregulated, and it is well established that multisensory neurons in the superior

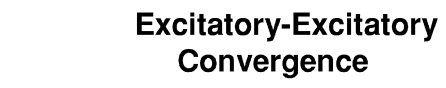

Excitatory-Inhibitory Convergence
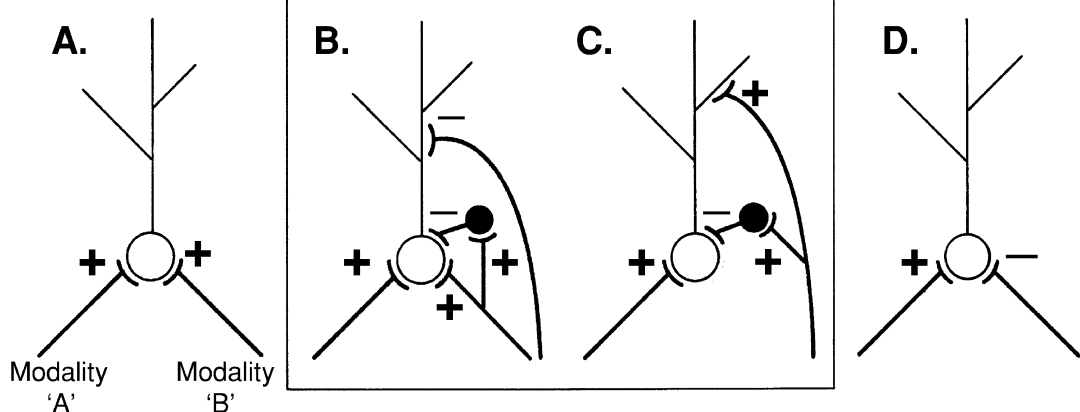

Fig. 6. Functional architecture of different patterns of multisensory convergence. Ideally, the results of multisensory convergence are dependent on the dominant inputs present, either as (A) excitatory inputs from both modality 'A' and modality 'B', or (D) as excitatory inputs from one with inhibitory inputs from the other. However, neural circuits are more complex than these representative schemes and, as indicated in the boxed area, most likely occur as blends between one extreme and the other. Current data indicate that multisensory neurons receiving 'excitatory-excitatory' convergence are also influenced by distant and local inhibitory projections (B), and 'excitatory-inhibitory' neurons are also likely to receive subthreshold excitatory inputs (C) from the 'inhibitory' modality. These observations suggest that the actual forms of multisensory convergence, of which there may be many, probably exist on a continuum between the two poles of 'excitatory-excitatory' and 'excitatory-inhibitory' convergence. 
colliculus also receive a variety of inhibitory inputs from extrinsic (for review, see Ref. [26]) as well as intrinsic sources $[38,43]$. Many deep layer bimodal neurons exhibit inhibitory receptive field surrounds $[31,40]$ and demonstrate multisensory response depression when the temporal $[37,45]$ or spatial relationships $[39,40]$ of the stimuli are out of alignment and are inhibited during local circuit activity controlled by specific behaviors $[1,38,43]$. Thus, there is compelling evidence for inhibitory signals that are carried perhaps as a component of the dominant excitatory projections to multisensory superior colliculus neurons. Therefore, perhaps a more accurate depiction of an 'excitatory-excitatory' convergence would include a lesser proportion of inhibitory inputs that assist in local circuit modulation and in the construction of modality-specific receptive fields. This modified representation of 'excitatory-excitatory' convergence might be as depicted in Fig. $6 \mathrm{~B}$, where excitation would play a dominant role and spatially/temporally coincident multiple sensory stimuli could generate response enhancement. Given that behaviorally-relevant multisensory stimuli are causally-related (e.g., the different 'energies' created from the impact of a falling rock; see Fig. 1), and these causally-related stimuli generally emanate from the same point in time and space (see Fig. 4), it is clear that this form of multisensory convergence is well suited for enhancing responses to these phenomena to facilitate detecting, localizing, and orienting/escape behaviors $[21,52,55]$. Also, because the signals from each of the convergent modalities are merged into an integrated and substantially transformed product, these effects are likely to have less to do with fine feature analysis of the stimuli than the presence/location of the stimuli in space. In addition, an 'excitatory-excitatory' form of convergence is likely to be incorporated into other multisensory circuits and perceptual processes, given that some multisensory effects, such as ventriloquism and 'cocktail party' effects, also have strong spatial and temporal attributes.

A second form of multisensory neuronal convergence, one involving an excitatory-inhibitory organization, is more novel, although its circuit design is well known within the modality-specific cortices (e.g., Refs. $[13,17,18,48,63])$. Convergent 'excitatory-inhibitory' multisensory circuits are likely to be more complex than simply the merging of their dominant effects in the manner depicted in Fig. 5D. At least for cortical circuits, the convergent inhibitory component probably is indirect. Because most cortico-cortical projection neurons are known to have a pyramidal morphology and are excitatory, it is seems likely that the observed effects occur indirectly via synapses on inhibitory interneurons. Furthermore, because of the cortical architecture involved, it is also probable that subthreshold excitatory inputs, perhaps onto distal dendrites, accompany the inhibitory inputs from the second modality (see Fig. 6C). Thus, spatially and temporally coincident stimuli would activate their respective modalities, but inputs from one modality would not enhance but inhibit those from the other. In this fashion, attention to a specific locus, but not to an anticipated modality, could lead to a suppressed response (e.g., Refs. $[25,57])$ that serves to differentiate attention toward the anticipated modality. Also, extinction of responses to a contralateral stimulus in one modality could be achieved through inhibition from an ipsilateral stimulus of the same or different modality [15,34], although the role of spatial disparity in these particular tests has not been completely ruled out. At a more general level, 'excitatory-inhibitory' convergence appears to act to modulate the activity of an excitatory modality through inhibition and disinhibition rather than express the more dramatic response changes observed during multisensory response enhancement. Ultimately, the subtlety of these modulatory effects may be better suited to influence the quality of a perception rather than to detect or localize an environmental event. In fact, under conditions of excitatory-inhibitory convergence, it would be predicted that spatially coincident stimuli would serve to depress (not enhance) multisensory response activity.

It is particularly important to note that, of the SIV neurons tested for cross-modal excitatory-inhibitory convergence, an overwhelming majority (nearly $70 \%$ ) were affected $[7,10]$. Furthermore, because the convergent inhibitory effects were evident only when the stimuli were combined, current extracellular sampling methods (using stimuli from different modalities but presented sequentially) will be ineffective in identifying excitatory-inhibitory multisensory convergence unless there is an unusually high and regular rate of spontaneous activity in the neurons examined. Thus, it is possible that the proportion of multisensory neurons (inclusive of excitatory-excitatory and excitatory-inhibitory types) in cortex is vastly underestimated.

\section{Other forms of multisensory convergence}

Even though the multisensory convergent circuits proposed above span a continuum between excitation and inhibition, this scheme is not intended as an exclusive representation of all possible multisensory convergence patterns. Certainly, other forms of multisensory convergence must exist. At least one modification to those proposed above is already known: within the superior colliculus, there is a subpopulation of 'excitatory-excitatory' multisensory neurons which fail to integrate multisensory responses [30] (Stein, personal communication). These neurons respond to inputs from more than one modality but their responses fail to significantly influence one another. These superior colliculus neurons appear to lack cortical inputs, which are critical for multisensory integrative capacities of superior colliculus neurons $[30,60,61]$. The mechanisms underlying these effects ap- 
pear to involve NMDA receptors, since cortical inputs to superior colliculus neurons involve NMDA-dependent components [5] and integrated multisensory responses in collicular neurons are dependent on NMDA-gated channels [4]. Therefore, excitatory inputs to multisensory neurons are complex and contain at least two functional modes: one which conveys excitatory inputs from a given modality and another, mediated through cortex, which augments excitation into an integrative level. Thus, it seems possible that the excitatory components of a modality-specific input may be mediated by different excitatory receptor classes or subclasses. How these various forms of inputs to superior colliculus neurons might contribute to the phenomena of multisensory processing will undoubtedly be the subject of continued interest and investigation. Nevertheless, what is already clear is that multisensory convergence is necessary but not sufficient in itself to generate multisensory response integration (e.g., response enhancement, response depression; Stein, personal communication).

The proposed patterns of multisensory convergence described above can account, in general terms, for the multisensory activity resulting from temporally coincident stimuli. However, it is entirely unknown how neural convergence patterns might underlie sequential multisensory events. For example, cross-modal matching (or sensory substitution) requires the formation of a neural percept or representation within one modality, and then converting that information in some fashion to represent or access another modality. While such tasks undoubtedly involve multisensory circuits that are substantially different than those proposed above, their actual configuration remains unresolved. Because the amygdala is necessary for crossmodal matching [44], an analysis of amygdalar multisensory circuitry and connectivity could provide fascinating insights into yet unknown forms of multisensory convergent circuitry.

\section{Conclusions}

Collectively, these observations and possibilities suggest that architecture of multisensory convergence occurs in at least two different functional forms: those representing convergence of two primarily excitatory inputs (excitatory-excitatory convergence) and those which result from a combination of excitatory afferents from one modality with inhibitory inputs from another (excitatory-inhibitory convergence) (Fig. 6). Each of these convergence patterns generates fundamentally different forms of multisensory interactions: excitatory-excitatory convergence produces dramatic response interactions in the form of multisensory response enhancement; excitatory-inhibitory convergence appears to produce more subtle modulations in the form of inhibition and disinhibition. It is possible that each of these different multisensory effects, determined by the nature of the convergence that produced them, ultimately contribute to distinct types of multisensory perceptions or behaviors.

\section{Acknowledgements}

Thanks to David Lewkowicz, whose perspective on multisensory perception helped initiate this inquiry into the nature of multisensory convergence. Thanks also to Ruth Clemo and Gyorgi Benedek for their insight and critical review of this manuscript. Supported by NIH grant NS39460.

\section{References}

[1] A.H. Bell, B.D. Corneil, M.A. Meredith, J. Van Opstal, V.C. Abrahams, D.P. Munoz, Multisensory integration in the superior colliculus of awake behaving primates, Soc. Neurosci. Abstr. 26 (2000) 1221.

[2] G. Benedek, T.P. Hicks, The visual insular cortex of the cat: organization, properties and modality specificity, Brain Res. 75 (1988) 271-277.

[3] A. Berman, Overlap of somatic and auditory cortical response fields in anterior ectosylvian gyrus of cat, J. Neurophysiol. 24 (1961) $608-620$.

[4] K.E. Binns, T.E. Salt, Importance of NMDA receptors for multimodal integration in the deep layers of the cat superior colliculus, J. Neurophysiol. 75 (1996) 920-930.

[5] K.E. Binns, T.E. Salt, Corticofugal influences on visual responses in cat superior colliculus: the role of NMDA receptors, Vis. Neurosci. 13 (1996) 683-694.

[6] L.M. Chalupa, R.W. Rhoades, Responses of visual, somatosensory, and auditory neurones in the golden hamster's superior colliculus, J. Physiol. (Lond.) 207 (1977) 595-626.

[7] H.R. Clemo, L.R. Dehner, M.A. Meredith, Cross-modal circuitry of anterior ectosylvian sulcal (AES) cortex: II. Functional connections from auditory Field AES to somatosensory SIV, Soc. Neurosci. Abstr. 26 (2000) 1221.

[8] H.R. Clemo, B.E. Stein, Somatosensory cortex: a 'new' somatotopic representation, Brain Res. 235 (1982) 162-168.

[9] H.R. Clemo, B.E. Stein, Organization of a fourth somatosensory area of cortex in cat, J. Neurophysiol. 50 (1983) 910-925.

[10] L.R. Dehner, H.R. Clemo, M.A. Meredith, Cross-modal circuitry of anterior ectosylvian sulcal (AES) cortex: I. Anatomical connections from auditory Field AES to somatosensory SIV, Soc. Neurosci. Abstr. 26 (2000) 1221.

[11] U.C. Drager, D.H. Hubel, Topography of visual and somatosensory projections in mouse superior colliculus, J. Neurophysiol. 39 (1976) 91-101.

[12] J.R. Duhamel, C.L. Colby, M.E. Goldberg, Congruent representations of visual and somatosensory space in single neurons of monkey ventral intraparietal cortex (Area VIP), in: J. Paillard (Ed.), Brain and Space, Oxford University Press, 1991, pp. 223-236.

[13] F.F. Ebner, M.A. Armstrong-James, Intracortical processes regulating the integration of sensory information, Prog. Brain Res. 86 (1990) 129-141.

[14] J.H. Fallon, L.A. Benevento, Auditory-visual interaction in cat orbital-insular cortex, Neurosci. Lett. 6 (1977) 143-149.

[15] A. Farne, F. Pavani, F. Meneghello, E. Ladavas, Left tactile extinction following visual stimulation of a rubber hand, Brain (in press).

[16] J.J. Foxe, G.V. Simpson, S.P. Ahlfors, Parieto-occipital $\sim 10 \mathrm{~Hz}$ 
activity reflects anticipatory state of visual attention mechanisms, NeuroReport 9 (1998) 3929-3933.

[17] C.D. Gilbert, Circuitry, architecture, and functional dynamics of visual cortex, Cereb. Cortex 3 (1993) 373-386.

[18] C.D. Gilbert, Adult cortical dynamics, Physiol. Rev. 78 (1998) 467-485.

[19] M.S.A. Graziano, C.G. Gross, A bimodal map of space: somatosensory receptive fields in the macaque putamen with corresponding visual receptive fields, Exp. Brain Res. 97 (1993) 96-109.

[20] M.S.A. Graziano, C.G. Gross, Visuospatial properties of ventral premotor cortex, J. Neurophysiol. 77 (1997) 2268-2292.

[21] D. Guitton, D.P. Munoz, Control of orienting gaze shifts by the tectoreticulospinal system in the head-free cat. I. Identification, localization, and effects of behavior on sensory responses, J. Neurophysiol. 66 (1991) 1605-1623.

[22] P.H. Hartline, L. Kass, M.S. Loop, Merging of modalities in the optic tectum: infrared and visual integration in rattlesnakes, Science 199 (1978) 1225-1229.

[23] J.K. Harting, S. Feig, D.P. Van Lieshout, Cortical somatosensory and trigeminal inputs to the cat superior colliculus: light and electron microscopic analyses, J. Comp. Neurol. 388 (1997) 313-326.

[24] T.P. Hicks, G. Benedek, G.A. Thurlow, Modality specificity of neuronal responses within the cat's insula, J. Neurophysiol. 60 (1988) 422-437.

[25] S.A. Hillyard, G.V. Simpson, D.L. Woods, S. VanVoorhis, T.F. Munte, Event-related brain potentials and selective attention to different modalities, in: F. Reinoso-Suarez, C. Ajmone-Marsan (Eds.), Cortical Integration, Raven, New York, 1984, pp. 395-415.

[26] M.F. Huerta, J.K. Harting, The mammalian superior colliculus: studies of its morphology and connections, in: H. Vanegas (Ed.), Comparative Neurology of the Optic Tectum, Plenum, New York, 1984, pp. 687-773.

[27] A. Iriki, M. Tanaka, Y. Iwamura, Coding of modified body schema during tool use by macaque postcentral neurones, NeuroReport 7 (1996) 2325-2330

[28] H. Jiang, F. Lepore, M. Pito, J.P. Guillemot, Sensory interactions in the anterior ectosylvian cortex of cats, Exp. Brain Res. 101 (1994) 385-396.

[29] W. Jiang, M.T. Wallace, H. Jiang, J.W. Vaughan, B.E. Stein, Two cortical areas mediate multisensory integration in superior colliculus neurons, J. Neurophysiol. 85 (2001) 506-522.

[30] E.G. Jones, T.P.S. Powell, An anatomical study of converging sensory pathways within the cerebral cortex of the monkey, Brain 93 (1970) 793-820.

[31] D.C. Kadunce, J.W. Vaughan, M.T. Wallace, G. Benedek, B.E. Stein, Mechanisms of within- and cross-modality suppression in the superior colliculus, J. Neurophysiol. 78 (1997) 2834-2847.

[32] A.J. King, A.R. Palmer, Cells responsive to free-field auditory stimuli in guinea-pig superior colliculus: distribution and response properties, J. Physiol. 342 (1983) 361-381.

[34] E. Ladavas, G. di Pellegrino, A. Farne, G. Zeloni, Neuropsychological evidence of an integrated visuotactile representation of peripersonal space in humans, J. Cogn. Neurosci. 10 (1998) 581-589.

[35] M.A. Meredith, H.R. Clemo, Auditory cortical projections from the anterior ectosylvian sulcus (Field AES) to the superior colliculus in cat: an anatomical and electrophysiological study, J. Comp. Neurol. 289 (1989) 687-707.

[36] M.A. Meredith, H.R. Clemo, B.E. Stein, The somatotopic component of the multisensory map in the deep laminae of the cat superior colliculus, J. Comp. Neurol. 312 (1991) 353-370.

[37] M.A. Meredith, J.W. Nemitz, B.E. Stein, Determinants of multisensory integration in superior colliculus neurons. I. Temporal factors, J. Neurosci. 7 (1987) 3215-3229.

[38] M.A. Meredith, A.S. Ramoa, Intrinsic circuitry of the superior colliculus: pharmacophysiological identification of horizontally-oriented inhibitory interneurons, J. Neurophysiol. 79 (1998) 15971602
[39] M.A. Meredith, B.E. Stein, Visual, auditory, and somatosensory convergence on cells in the superior colliculus results in multisensory integration, J. Neurophysiol. 56 (1986) 640-662.

[40] M.A. Meredith, B.E. Stein, Spatial determinants of multisensory integration in cat superior colliculus neurons, J. Neurophsyiol. 75 (1996) 1843-1857.

[41] J.C. Middlebrooks, E.I. Knudsen, A neural code for auditory space in the cat's superior colliculus, J. Neurosci. 4 (1984) 2621-2634.

[42] J. Moran, R. Desimone, Selective attention gates visual processing in the extrastriate cortex, Science 229 (1985) 782-784.

[43] D.P. Munoz, P.J. Istvan, Lateral inhibitory interactions in the intermediate layers of the monkey superior colliculus, J. Neurophysiol. 79 (1998) 1193-1209.

[44] E.A. Murray, M. Mishkin, Amygdalectomy impairs crossmodal association in monkeys, Science 228 (1985) 604-606.

[45] S. Quessy, A.J. Sweatt, B.E. Stein, T.R. Stanford, The influence of stimulus intensity and timing on the responses of multisensory neurons in the superior colliculus: comparison to a model's prediction, Soc. Neurosci. Abstr. 30 (2000) 1221.

[46] R. Ramachandran, M.T. Wallace, H.R. Clemo, B.E. Stein, Multisensory convergence and integration in rat cortex, Soc. Neurosci. Abstr. 19 (1993) 1447

[47] J.H. Reynolds, L. Chelazzi, R. Desimone, Competitive mechanisms subserve attention in macaque areas V2 and V4, J. Neurosci. 19 (1999) 1736-1753.

[48] K. Richter, A. Hess, H. Scheich, Functional mapping of transsynaptic effects of local manipulation of inhibition in gerbil auditory cortex, Brain Res. 831 (1999) 184-199.

[49] M. Sams, R. Aulanko, M. Hamalainen, R. Hari, O.V. Lounasmaa, S.T. Lu, J. Simola, Seeing speech: visual information from lip movements modified activity in the human auditory cortex, Neurosci. Lett. 127 (1991) 141-145

[50] C.E. Schroeder, R.W. Lindsley, C. Specht, A. Marcovici, J.F. Smiley, D.C. Javitt, Somatosensory input to auditory association cortex in the macaque monkey, J. Neurophysiol. 85 (2001) 13221327.

[51] B. Selzer, D.N. Pandya, Parietal, temporal, and occipital projections to cortex of the superior temporal sulcus in the rhesus monkey: a retrograde tracer study, J. Comp. Neurol. 343 (1994) 445-463.

[52] B.E. Stein, W.S. Huneycutt, M.A. Meredith, Neurons and behavior: the same rules of multisensory integration apply, Brain Res. 448 (1988) 355-357.

[53] B.E. Stein, B. Magalhaes-Castro, L. Kruger, Relationship between visual and tactile representation in cat superior colliculus, J. Neurophysiol. 39 (1976) 401-419.

[54] B.E. Stein, M.A. Meredith, Merging of the Senses, MIT Press, Cambridge, 1993.

[55] B.E. Stein, M.A. Meredith, W.S. Huneycutt, L.W. McDade, Behavioral indices of multisensory integration: orientation top visual cues is affected by auditory stimuli, J. Cogn. Neurosci. 1 (1989) $12-24$.

[56] B.E. Stein, M.A. Meredith, M.T. Wallace, The visually responsive neurons and beyond: multisensory integration in cat and monkey, Prog. Brain Res. 95 (1993) 79-90.

[57] W.A. Teder-Salejarvi, T.F. Munte, F. Sperlich, S.C. Hillyard, Intramodal and cross-modal spatial attention to auditory and visual stimuli: an event-related brain potential study, Cogn. Brain Res. 8 (1999) 327-343.

[58] Y.-C. Tiao, C. Blakemore, Functional organization in the superior colliculus of the golden hamster, J. Comp. Neurol. 168 (1976) 483-506.

[59] M.T. Wallace, M.A. Meredith, B.E. Stein, The integration of multiple sensory inputs in cat cortex, Exp. Brain Res. 91 (1992) 484-488.

[60] M.T. Wallace, M.A. Meredith, B.E. Stein, Converging influences from visual, auditory, and somatosensory cortices onto output neurons of the superior colliculus, J. Neurophysiol. 69 (1993) $1797-1809$ 
[61] M.T. Wallace, B.E. Stein, Cross-modal synthesis in the midbrain depends on input from cortex, J. Neurophysiol. 71 (1994) 429-432.

[62] M.T. Wallace, L.K. Wilkinson, B.E. Stein, Representation and integration of multiple sensory inputs in primate superior colliculus, J. Neurophysiol. 76 (1996) 1246-1266.

[63] J. Wang, D. Caspary, R.J. Salvi, GABA-A antagonist causes dramatic expansion of tuning in primary auditory cortex, NeuroReport 11 (2000) 1137-1140.

[64] J.G. Wespic, Multimodal sensory activation of cells in the magnocellular medial geniculate nucleus, Exp. Neurol. 15 (1966) 299-318.

[65] L.Z. Wise, D.R.F. Irvine, Topographic organization of interaural intensity difference sensitivity in deep layers of cat superior colliculus: implications for auditory spatial representation, J. Neurophysiol. 54 (1985) 185-211.

[66] M.S. Worden, J.J. Foxe, N. Wang, G.V. Simpson, Anticipatory biasing of visuospatial attention indexed by retinotopically specificband electroencephalography increases over occipital cortex, J. Neurosci. 20 (2000) RC63.

[67] S. Zangenehpour, A. Chaudhuri, Multisensory neurons of the superior colliculus revealed by dual fluorescent ICC/ISH labeling using c-fos and zif268 expression profiles, Soc. Neurosci. Abstr. 29 (1999) 1414. 